\title{
Hofstadter point spectrum trace and the almost Mathieu operator
}

Cite as: J. Math. Phys. 59, 073504 (2018); https://doi.org/10.1063/1.5020147

Submitted: 20 December 2017 . Accepted: 21 June 2018 . Published Online: 12 July 2018

Stéphane Ouvry, Stephan Wagner, and Shuang Wu (D)
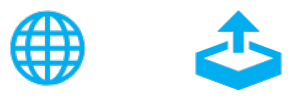

\section{ARTICLES YOU MAY BE INTERESTED IN}

Zeros of rational functions and solvable nonlinear evolution equations

Journal of Mathematical Physics 59, 072701 (2018); https://

doi.org/10.1063/1.5033543

Real homotopy theory and supersymmetric quantum mechanics

Journal of Mathematical Physics 59, 072102 (2018); https://

doi.org/10.1063/1.5011677

Hofstadter's butterfly and Langlands duality

Journal of Mathematical Physics 59, 061704 (2018); https://

doi.org/10.1063/1.4998635

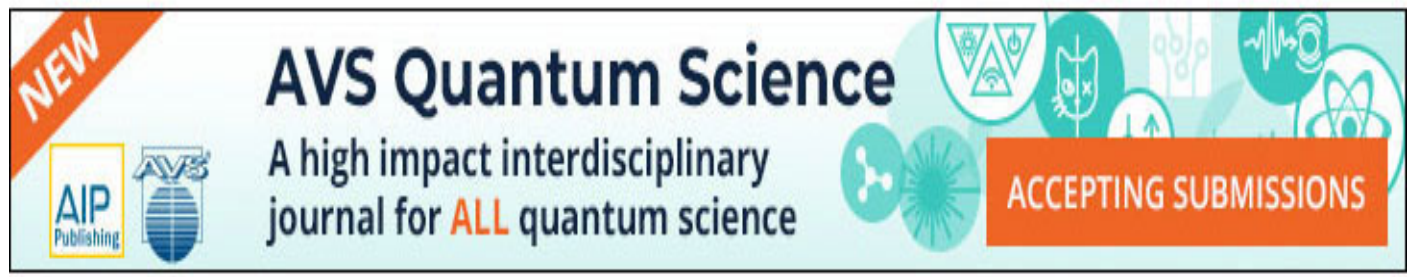




\title{
Hofstadter point spectrum trace and the almost Mathieu operator
}

\author{
Stéphane Ouvry, ${ }^{1}$ Stephan Wagner, ${ }^{2}$ and Shuang $\mathrm{Wu}^{1}$ \\ ${ }^{1}$ LPTMS, CNRS, Université Paris-Sud, Université Paris-Saclay, 91405 Orsay Cedex, France \\ ${ }^{2}$ Department of Mathematical Sciences, Stellenbosch University, Matieland, \\ Stellenbosch 7602, South Africa
}

(Received 20 December 2017; accepted 21 June 2018; published online 12 July 2018)

\begin{abstract}
We consider point spectrum traces in the Hofstadter model. We show how to recover the full quantum Hofstadter trace by integrating these point spectrum traces with the appropriate free density of states on the lattice. This construction is then generalized to the almost Mathieu operator and its $n$th moments which can be expressed in terms of generalized Kreft coefficients. Published by AIP Publishing. https://doi.org/10.1063/1.5020147
\end{abstract}

\section{INTRODUCTION}

In Ref. 1, we focused on the algebraic area generating function of closed lattice walks of a given length $n$ ( $n$ is then necessarily even),

$$
Z_{n}\left(e^{i \gamma}\right)
$$

evaluated at $e^{i \gamma}$, a root of unity. One reason for studying this quantity arises from the connection of the algebraic area distribution of random curves to the quantum spectrum of a charged particle in a perpendicular magnetic field. In the lattice case at hand, the mapping is on the quantum Hofstadter model $^{2}$ of a particle hopping on a two-dimensional lattice in a magnetic flux $\gamma$, counted in unit of the flux quantum. More precisely, $Z_{n}\left(e^{i \gamma}\right)$ is mapped on the $n$th moment $\operatorname{Tr} H_{\gamma}^{n}$ of the Hofstadter Hamiltonian $\mathrm{H}_{\gamma}$-thereafter referred to as the quantum Hofstadter trace-

$$
Z_{n}\left(e^{i \gamma}\right)=\operatorname{Tr} H_{\gamma}^{n}
$$

by virtue of which evaluating $Z_{n}\left(e^{i \gamma}\right)$ for classical lattice walks gives an expression ${ }^{1}$ for the Hofstadter quantum trace $\operatorname{Tr} H_{\gamma}^{n}$. In the simplified case of a rational flux, not surprisingly, the trace $\operatorname{Tr} H_{\gamma}^{n}$ can be written in terms of the Kreft coefficients ${ }^{3}$ which encode the Schrödinger equation for the Hofstadter model

$$
\Phi_{m+1}+\Phi_{m-1}+2 \cos \left(k_{y}+\gamma m\right) \Phi_{m}=E \Phi_{m} .
$$

One would like to generalize this construction to the almost Mathieu operator case

$$
\Phi_{m+1}+\Phi_{m-1}+\lambda \cos \left(k_{y}+\gamma m\right) \Phi_{m}=E \Phi_{m},
$$

where $\lambda$ is now a free parameter. This operator, among other things, plays an important role in the characterization of the fractal structure of the Hofstadter spectrum. ${ }^{4}$ Physically, it describes a quantum particle hopping on a lattice with horizontal and vertical amplitudes in a ratio $\lambda$.

We will first rederive the results of Ref. 1 by starting from point spectrum traces-to be defined later-and integrating them with the appropriate free density of states on the lattice in order to recover the quantum trace $\operatorname{Tr} H_{\gamma}^{n}$. This approach is original and gives a new light on the results obtained in Ref. 1. The generalization to the almost Mathieu case will then follow provided that one can extend accordingly the Kreft coefficients construction to the $\lambda \neq 2$ case. Finally we will discuss in Sec. V some direct links which can be established with current activities in the field. ${ }^{10}$ 


\section{A REMINDER}

In the commensurate case with a rational flux $\gamma=2 \pi p / q$, with $p$ and $q$ co-primes, the lattice Hofstadter eigenstates $\psi_{m, n}=e^{i n k_{y}} \Phi_{m}$ are $q$-periodic on the horizontal axis $\Phi_{m+q}=e^{i q k_{x}} \Phi_{m}$. The Schrödinger equation (1) then reduces to a $q \times q$ secular matrix $m_{p / q}\left(E, k_{x}, k_{y}\right)$ acting with zero output on the $q$-components eigenvector $\left\{\Phi_{0}, \Phi_{1}, \ldots, \Phi_{q-1}\right\}$,

$$
\left(\begin{array}{cccccc}
2 \cos \left(k_{y}\right)-E & 1 & 0 & \cdots & 0 & e^{-i q k_{x}} \\
1 & 2 \cos \left(k_{y}+\frac{2 \pi p}{q}\right)-E & 1 & \cdots & 0 & 0 \\
0 & 1 & () & \cdots & 0 & 0 \\
\vdots & \vdots & \vdots & \ddots & \vdots & \vdots \\
0 & 0 & 0 & \cdots & () & 1 \\
e^{i q k_{x}} & 0 & 0 & \cdots & 1 & 2 \cos \left(k_{y}+(q-1) \frac{2 \pi p}{q}\right)-E
\end{array}\right)\left(\begin{array}{c}
\Phi_{0} \\
\Phi_{1} \\
\Phi_{2} \\
\vdots \\
\Phi_{q-2} \\
\Phi_{q-1}
\end{array}\right)=\left(\begin{array}{c}
0 \\
0 \\
0 \\
\vdots \\
0 \\
0
\end{array}\right) .
$$

The $q$ eigenenergies $E_{1}\left(k_{x}, k_{y}\right), E_{2}\left(k_{x}, k_{y}\right), \ldots, E_{q}\left(k_{x}, k_{y}\right)$ are the $\operatorname{roots} \operatorname{of} \operatorname{det}\left(m_{p / q}\left(E, k_{x}, k_{y}\right)\right)=0$, which, thanks to the identity

$$
\operatorname{det}\left(m_{p / q}\left(E, k_{x}, k_{y}\right)\right)=\operatorname{det}\left(m_{p / q}(E, 0,0)\right)-2(-1)^{q}\left(\cos \left(q k_{x}\right)-1+\cos \left(q k_{y}\right)-1\right),
$$

rewrites (see Ref. 5) as

$$
\operatorname{det}\left(m_{p / q}(E, 0,0)\right)=2(-1)^{q}\left(\cos \left(q k_{x}\right)-1+\cos \left(q k_{y}\right)-1\right) .
$$

The polynomial $b_{p / q}(z)$ with coefficients $-a_{p / q}(2 j)$,

$$
b_{p / q}(z):=-\sum_{j=0}^{\left[\frac{q}{2}\right]} a_{p / q}(2 j) z^{2 j},
$$

$\left(a_{p / q}(0)=-1\right)$ materializes in $\operatorname{det}\left(m_{p / q}(E, 0,0)\right)$ as

$$
\operatorname{det}\left(m_{p / q}(E, 0,0)\right)+4(-1)^{q}=(-1)^{q} E^{q} b_{p / q}(1 / E)
$$

so that (5) becomes

$$
E^{q} b_{p / q}(1 / E)=2\left(\cos \left(q k_{x}\right)+\cos \left(q k_{y}\right)\right) .
$$

The $a_{p / q}(2 j)$ 's in $b_{p / q}(z)$ in (6) are related to the Kreft coefficients $\mathrm{c}_{p / q}(2 j)$ as

$$
\operatorname{det}\left(m_{p / q}(E, 0,0)\right)+4(-1)^{q}=\sum_{j=\frac{q}{2}-\left\lfloor\frac{q}{2}\right\rfloor}^{\frac{q}{2}} \mathrm{c}_{p / q}(2 j) E^{2 j}
$$

so that $a_{p / q}(2 j)=\mathrm{c}_{p / q}(q-2 j)(-1)^{q+1}$. One gets

$$
\begin{aligned}
a_{p / q}(2 j)= & (-1)^{j+1} \sum_{k_{1}=0}^{q-2 j} \sum_{k_{2}=0}^{k_{1}} \ldots \sum_{k_{j}=0}^{k_{j-1}} 4 \sin ^{2}\left(\frac{\pi\left(k_{1}+2 j-1\right) p}{q}\right) 4 \sin ^{2}\left(\frac{\pi\left(k_{2}+2 j-3\right) p}{q}\right) \ldots \\
& \times 4 \sin ^{2}\left(\frac{\pi\left(k_{j}+1\right) p}{q}\right),
\end{aligned}
$$

with building blocks

$$
4 \sin ^{2}\left(\frac{\pi(k+1) p}{q}\right)=e^{-i k_{y}}\left(1-e^{\frac{2 i \pi(k+1) p}{q}}\right) e^{i k_{y}}\left(1-e^{-\frac{2 i \pi(k+1) p}{q}}\right)=\alpha_{p / q}(k) \bar{\alpha}_{p / q}(k),
$$

where

$$
\alpha_{p / q}(k)=e^{-i k_{y}}\left(1-e^{\frac{2 i \pi(k+1) p}{q}}\right)
$$


and $\bar{\alpha}_{p / q}(k)$ is the complex conjugate. How to derive (9) is explained in Kreft's paper $^{3}$ (see the Appendix for details). Note that $a_{p / q}(2 j)=0$ as soon as $q<2 j$.

A closed expression for the quantum Hofstadter trace, which is defined as

$$
\operatorname{Tr} H_{2 \pi p / q}^{n}=\frac{1}{q} \int_{-\pi}^{\pi} \int_{-\pi}^{\pi} \frac{d k_{x}}{2 \pi} \frac{d k_{y}}{2 \pi} \sum_{r=1}^{q} E_{r}^{n}\left(k_{x}, k_{y}\right),
$$

where one has integrated over the quasi-momenta $k_{x}$ and $k_{y} \in[-\pi, \pi]$ the sum of the $q$ eigenenergies $E_{r}\left(k_{x}, k_{y}\right)$ - the roots of (8)-at a power $n$, has been obtained ${ }^{1}$ in terms of the Kreft coefficients

$$
\begin{aligned}
\operatorname{Tr} H_{2 \pi p / q}^{n}= & \frac{n}{q} \sum_{k \geq 0} \sum_{\begin{array}{c}
\ell_{1}, \ell_{2}, \ldots, \ell_{\lfloor q / 2\rfloor} \geq 0 \\
\ell_{1}+2 \ell_{2}+\cdots+\lfloor q / 2\rfloor \ell_{\lfloor q / 2\rfloor}=n / 2-q k
\end{array}} \frac{\left(\begin{array}{c}
\ell_{1}+\ell_{2}+\cdots+\ell_{\lfloor q / 2\rfloor}+2 k \\
\ell_{1}, \ell_{2}, \ldots, \ell_{\lfloor q / 2\rfloor}, 2 k
\end{array}\right)}{\ell_{1}+\ell_{2}+\cdots+\ell_{\lfloor q / 2\rfloor}+2 k}\left(\begin{array}{c}
2 k \\
k
\end{array}\right)^{2} \\
& \prod_{j=1}^{\lfloor q / 2\rfloor} a_{p / q}(2 j)^{\ell_{j}},
\end{aligned}
$$

with the generating function

$$
\sum_{n \geq 0} \operatorname{Tr} H_{2 \pi p / q}^{n} z^{n}=\left(1-\frac{z b_{p / q}^{\prime}(z)}{q b_{p / q}(z)}\right) \frac{2}{\pi} K\left(\frac{16 z^{2 q}}{b_{p / q}(z)^{2}}\right),
$$

where $K$ is the complete elliptic integral of the first kind, $\frac{2}{\pi} K(16 x)=\sum_{k \geq 0}\left(\begin{array}{c}2 k \\ k\end{array}\right)^{2} x^{k}$.

\section{POINT SPECTRUM TRACE FORMULA AND DENSITY OF STATES}

\section{A. Mid-band trace formula}

One aims at generalizing (11) and (12) to the almost Mathieu operator case (2). To achieve this goal, first of all, one remarks that the Hofstadter trace (11), valid for all $n$ and $q$, coincides when $q$ $>n / 2$ with the mid-band traces given for the particular cases $n=2, \ldots, 10$ in Ref. 6 . The mid-band trace

$$
\operatorname{Tr}_{0} H_{2 \pi p / q}^{n}=\frac{1}{q} \sum_{r=1}^{q} E_{r}^{n}(0),
$$

to be distinguished from the quantum Hofstadter trace (10), is taken solely on the mid-band energies $E_{r}(0)$, the roots of Ref. 7

$$
E^{q} b_{p / q}(1 / E)=0,
$$

to be distinguished from the $E_{r}\left(k_{x}, k_{y}\right)$ 's, the roots of (8) (for an illustration see Figs. 1 and 2).

It is quite straightforward to obtain for all $n$ and $q$ the mid-band trace formula

$$
\operatorname{Tr}_{0} H_{2 \pi p / q}^{n}=\frac{n}{q} \sum_{\substack{\ell_{1}, \ell_{2}, \ldots, \ell_{\lfloor q / 2\rfloor} \geq 0 \\
\ell_{1}+2 \ell_{2}+\cdots+\lfloor q / 2\rfloor \ell_{\lfloor q / 2\rfloor}=n / 2}} \frac{\left(\begin{array}{c}
\ell_{1}+\ell_{2}+\cdots+\ell_{\lfloor q / 2\rfloor} \\
\ell_{1}, \ell_{2}, \ldots, \ell_{\lfloor q / 2\rfloor}
\end{array}\right)}{\ell_{1}+\ell_{2}+\cdots+\ell_{\lfloor q / 2\rfloor}} \prod_{j=1}^{\lfloor q / 2\rfloor} a_{p / q}(2 j)^{\ell_{j}}
$$

One can check that the Hofstadter trace (11) and the mid-band trace (14) indeed coincide provided that $q>n / 2$ since then the summation index $k$ in (11) necessarily vanishes. A qualitative interpretation for this fact can stem from the classical picture of lattice walks contained in the quantum periodic cell of horizontal length $q$ (the lattice walk the farthest on the horizontal axis from the origin indeed goes to a distance $n / 2)$.

The mid-band trace (14) has a simple combinatorial interpretation: it is the sum of products of the $a_{p / q}(2 j)^{\ell_{j}}$,s corresponding to partitions of $n$ in even integer parts of size $2 j$ [no larger than $q$ since $a_{p / q}(2 j)=0$ as soon as $\left.q<2 j\right]$, i.e., $2 \ell_{1}+4 \ell_{2}+\cdots+2\lfloor q / 2\rfloor \ell_{\lfloor q / 2\rfloor}=n$, multiplied by the multinomial weight. 


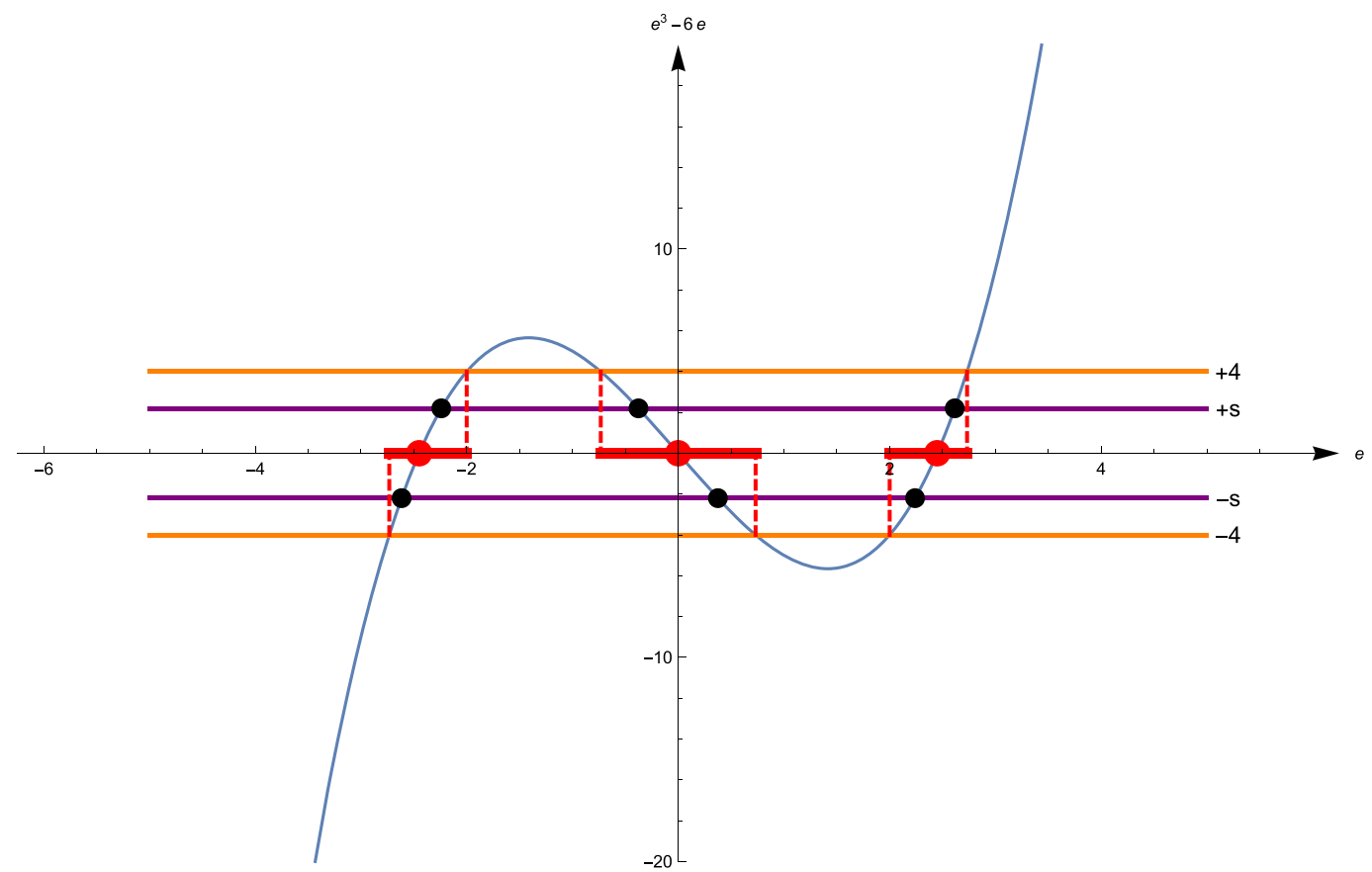

FIG. 1. $p=1, q=3: E^{q} b_{p / q}(1 / E)=E^{3}-6 E$; the 3 horizontal red segments are the energy bands; the 3 red dots are the mid-band energies; the 6 black dots are the $\pm s$ energies.

The generating function follows as

$$
\sum_{n \geq 0, n \text { even }} \operatorname{Tr}_{0} H_{2 \pi p / q}^{n} z^{n}=1-\frac{z b_{p / q}^{\prime}(z)}{q b_{p / q}(z)},
$$

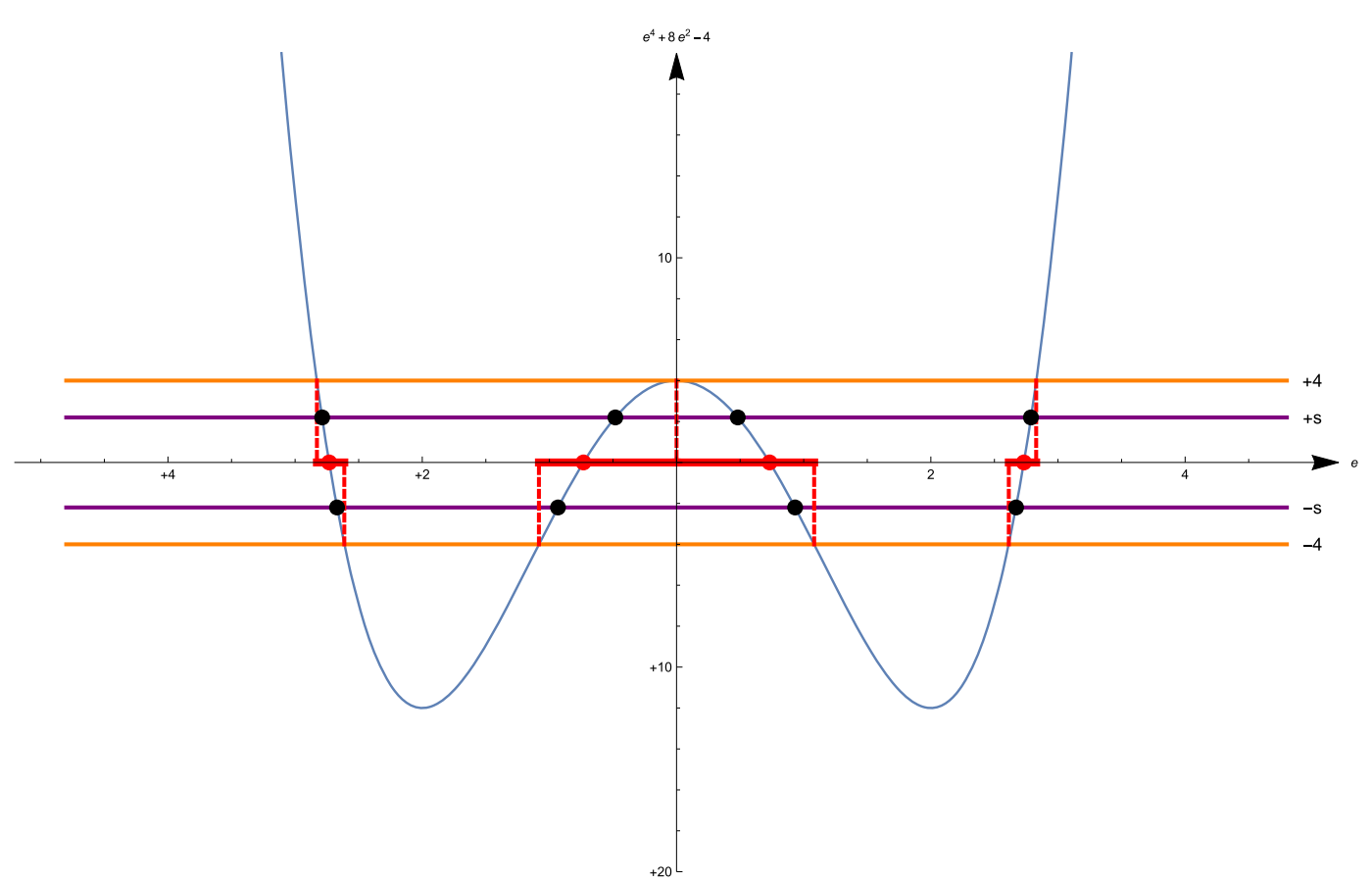

FIG. 2. $p=1, q=4: E^{q} b_{p / q}(1 / E)=E^{4}-8 E^{2}+4$; the 4 horizontal red segments are the energy bands; the 4 red dots are the mid-band energies; the 8 black dots are the $\pm s$ energies. 
which amounts to nothing else but (13); i.e., the $E_{r}(0)$ 's are the roots of $E^{q} b_{b / q}(1 / E)=0$. To see this, rewrite

$$
\begin{aligned}
\sum_{n \geq 0, n \text { even }} \operatorname{Tr}_{0} H_{2 \pi p / q}^{n} z^{n} & =\sum_{n \geq 0, n \text { even }} \frac{1}{q}\left(\sum_{r=1}^{q} E_{r}^{n}(0)\right) z^{n}=\frac{1}{q} \sum_{r=1}^{q} \sum_{n \geq 0, n \text { even }}\left(z E_{r}(0)\right)^{n} \\
& =\frac{1}{q} \sum_{r=1}^{q} \frac{1}{1-z^{2} E_{r}^{2}(0)}=\frac{1}{2 q} \sum_{r=1}^{q}\left(\frac{1}{1-z E_{r}(0)}+\frac{1}{1+z E_{r}(0)}\right)=\frac{1}{q} \sum_{r=1}^{q} \frac{1}{1-z E_{r}(0)}
\end{aligned}
$$

so that (15) becomes

$$
\frac{1}{q} \sum_{r=1}^{q} \frac{1}{1-z E_{r}(0)}=1-\frac{z b_{p / q}^{\prime}(z)}{q b_{p / q}(z)} .
$$

On the other hand, (13) rewrites as

$$
(1 / z)^{q} b_{p / q}(z)=\prod_{r=1}^{q}\left(1 / z-E_{r}(0)\right)
$$

that is,

$$
b_{p / q}(z)=\prod_{r=1}^{q}\left(1-z E_{r}(0)\right)
$$

so that

$$
\left(\log \left(b_{p / q}(z)\right)\right)^{\prime}=\sum_{r=1}^{q} \frac{-E_{r}(0)}{1-z E_{r}(0)}=\frac{1}{z} \sum_{r=1}^{q}\left(1-\frac{1}{1-z E_{r}(0)}\right)
$$

or

$$
\frac{b_{p / q}(z)^{\prime}}{b_{p / q}(z)}=\frac{1}{z}\left(q-\sum_{r=1}^{q} \frac{1}{1-z E_{r}(0)}\right)
$$

which is (16).

\section{B. $\pm s$ trace formula}

More generally, one can consider the $\pm s$ spectrum traces

$$
\operatorname{Tr}_{ \pm s} H_{2 \pi p / q}^{n}=\frac{1}{2 q}\left(\sum_{r=1}^{q} E_{r}^{n}(s)+\sum_{r=1}^{q} E_{r}^{n}(-s)\right)
$$

taken on the $2 q$ roots $E_{r}(s)$ and $E_{r}(-s)$ of

$$
E^{q} b_{p / q}(1 / E)=s \text { and } E^{q} b_{p / q}(1 / E)=-s
$$

with $-4 \leq s \leq 4$ since $-4 \leq 2\left(\cos \left(q k_{x}\right)+\cos \left(q k_{y}\right)\right) \leq 4$ in (8). The mid-band spectrum is obtained for $s=0$, and the edge-band spectrum is obtained for $s=4$, which is of particular interest as well (see Figs. 1 and 2).

Following the same line of reasoning as for the mid-band spectrum, one obtains the $\pm s$ spectrum trace generating function

$$
\begin{aligned}
\sum_{n \geq 0, n \text { even }} \operatorname{Tr}_{ \pm s} H_{2 \pi p / q}^{n} z^{n} & =1-\frac{z\left(b_{p / q}(z)-s z^{q}\right)^{\prime}}{2 q\left(b_{p / q}(z)-s z^{q}\right)}-\frac{z\left(b_{p / q}(z)+s z^{q}\right)^{\prime}}{2 q\left(b_{p / q}(z)+s z^{q}\right)} \\
& =1-\frac{z\left(b_{p / q}^{2}(z)-s^{2} z^{2 q}\right)^{\prime}}{2 q\left(b_{p / q}^{2}(z)-s^{2} z^{2 q}\right)} \\
& =\left(1-\frac{z b_{p / q}^{\prime}(z)}{q b_{p / q}(z)}\right) \frac{1}{1-s^{2}\left(\frac{z^{q}}{b_{p / q}(z)}\right)^{2}}
\end{aligned}
$$


which rightly reduces to the mid-band case (15) when $s=0$. Again (18) is a mere rewriting of the $E_{r}(s)$ 's and $E_{r}(-s)$ 's being the roots of $E^{q} b_{p / q}(1 / E)= \pm s$ in (17).

One has likewise the $\pm s$ trace formula

$$
\operatorname{Tr}_{ \pm s} H_{2 \pi p / q}^{n}=\frac{n}{q} \sum_{k \geq 0} \sum_{\begin{array}{c}
\ell_{1}, \ell_{2}, \ldots, \ell_{\lfloor q / 2\rfloor} \geq 0 \\
\ell_{1}+2 \ell_{2}+\cdots+\lfloor q / 2\rfloor \ell_{\lfloor q / 2\rfloor}=n / 2-q k
\end{array}} \frac{\left(\begin{array}{c}
\ell_{1}+\ell_{2}+\cdots+\ell_{\lfloor q / 2\rfloor}+2 k \\
\ell_{1}, \ell_{2}, \ldots, \ell_{\lfloor q / 2\rfloor}, 2 k
\end{array}\right)}{\ell_{1}+\ell_{2}+\cdots+\ell_{\lfloor q / 2\rfloor}+2 k} s k \prod_{j=1}^{\lfloor q / 2\rfloor} a_{p / q}(2 j)^{\ell_{j}},
$$

which again reduces to the mid-band trace (14) when $s=0$. The combinatorial interpretation of (19) is again simple in terms of products of $s^{2}$ and of the $a_{p / q}(2 j)$ 's corresponding to all partitions of $n$ in even integer parts of size $2 q$ and $2 j$, respectively.

Again, as soon as $q>n / 2$, the trace $\operatorname{Tr}_{ \pm s} H_{2 \pi p / q}^{n}$ in (19) does not depend on $s$ anymore and coincides with the Hofstadter trace (11) and, by the same token, with the mid-band trace (14).

\section{Density of states}

One remarks next that the Hofstadter quantum trace $\operatorname{Tr} H_{2 \pi p / q}^{n}$ in (11) can be recovered from the $\pm s$ spectrum trace $\operatorname{Tr}_{ \pm s} H_{2 \pi p / q}^{n}$ in (19) if one replaces $s^{2 k}$ by $\left(\begin{array}{c}2 k \\ k\end{array}\right)^{2}$. This replacement amounts to an integration of (19) over $s \in[-4,4]$ with the density of state $\rho(s)$,

$$
\int_{-4}^{4} \operatorname{Tr}_{ \pm s} H_{2 \pi p / q}^{n} \rho(s) d s=\operatorname{Tr} H_{2 \pi p / q}^{n}
$$

such that

$$
\int_{-4}^{4} s^{2 k} \rho(s) d s=\left(\begin{array}{c}
2 k \\
k
\end{array}\right)^{2}
$$

Clearly the free density of states on the $2 \mathrm{~d}$ lattice,

$$
\rho(s)=1 /\left(2 \pi^{2}\right) K\left(1-s^{2} / 16\right),
$$

enforces (21): it is the density of states for the spectrum $s=2\left(\cos \left(q k_{x}\right)+\cos \left(q k_{y}\right)\right)$. Again, as soon as $q>n / 2$, the integration in (20) becomes trivial, i.e., the identity. Note that in the spirit of Ref. 8 , one can make the change of variable $s= \pm E^{q} b_{p / q}(1 / E)$ in (21) to obtain

$$
\int_{-4}^{4}\left(E^{q} b_{p / q}(1 / E)\right)^{2 k} \rho_{p / q}(E) d E=\left(\begin{array}{c}
2 k \\
k
\end{array}\right)^{2},
$$

where $\rho_{p / q}(E)$ is the Hofstadter density of states. This yields the trace sum rule

$$
\operatorname{Tr}\left(H_{2 \pi p / q}^{q} b_{p / q}\left(1 / H_{2 \pi p / q}\right)\right)^{2 k}=\left(\begin{array}{c}
2 k \\
k
\end{array}\right)^{2}
$$

or

$$
\operatorname{Tr}\left(\sum_{j=0}^{\left[\frac{q}{2}\right]} a_{p / q}(2 j) H_{2 \pi p / q}^{q-2 j}\right)^{2 k}=\left(\begin{array}{c}
2 k \\
k
\end{array}\right)^{2}
$$

which is an inversion of (11).

For the generating function as well, which is given by

$$
\int_{-4}^{4} \sum_{n} \operatorname{Tr}_{ \pm s} H_{2 \pi p / q}^{n} z^{n} \rho(s) d s=\sum_{n} \operatorname{Tr} H_{2 \pi p / q}^{n} z^{n},
$$

one uses (12), (18), and (22) so that

$$
\int_{-4}^{4}\left(1-\frac{z\left(b_{p / q}^{2}(z)-s^{2} z^{2 q}\right)^{\prime}}{2 q\left(b_{p / q}^{2}(z)-s^{2} z^{2 q}\right)}\right) \frac{1}{2 \pi^{2}} K\left(1-\frac{s^{2}}{16}\right) d s=\left(1-\frac{z b_{p / q}^{\prime}(z)}{q b_{p / q}(z)}\right) \frac{2}{\pi} K\left(16\left(\frac{z^{q}}{b_{p / q}(z)}\right)^{2}\right)
$$

has to be satisfied. After simplification, this narrows down to 


$$
\int_{-4}^{4} \frac{1}{2 \pi^{2}} K\left(1-\frac{s^{2}}{16}\right) \frac{1}{1-s^{2}\left(\frac{z^{q}}{b_{p / q}(z)}\right)^{2}} d s=\frac{2}{\pi} K\left(16\left(\frac{z^{q}}{b_{p / q}(z)}\right)^{2}\right),
$$

i.e.,

$$
\int_{-4}^{4} K\left(1-\frac{s^{2}}{16}\right) \frac{1}{1-s^{2} z^{2}} d s=4 \pi K\left(16 z^{2}\right)
$$

which is nothing but the complete elliptic integral of the first kind being the generating function for the square of the binomial coefficients, i.e., $\frac{2}{\pi} K\left(16 z^{2}\right)=\sum_{k}\left(\begin{array}{c}2 k \\ k\end{array}\right)^{2} z^{2 k}$.

\section{IV. $\lambda \neq 2$ : POINT SPECTRUM TRACE FORMULA AND DENSITY OF STATES}

All these considerations can be extended when $\lambda \neq 2$ to the almost Mathieu case (2) whose secular matrix is

$$
m_{p / q}^{(\lambda)}\left(E, k_{x}, k_{y}\right)=\left(\begin{array}{cccccc}
\lambda \cos \left(k_{y}\right)-E & 1 & 0 & \cdots & 0 & e^{-i q k_{x}} \\
1 & \lambda \cos \left(k_{y}+\frac{2 \pi p}{q}\right)-E & 1 & \cdots & 0 & 0 \\
0 & 1 & () & \cdots & 0 & 0 \\
\vdots & \vdots & \vdots & \ddots & \vdots & \vdots \\
0 & 0 & 0 & \cdots & () & 1 \\
e^{i q k_{x}} & 0 & 0 & \cdots & 1 & \lambda \cos \left(k_{y}+(q-1) \frac{2 \pi p}{q}\right)-E
\end{array}\right) .
$$

Thanks to the identity

$$
\operatorname{det}\left(m_{p / q}^{(\lambda)}\left(E, k_{x}, k_{y}\right)\right)=\operatorname{det}\left(m_{p / q}^{(\lambda)}(E, 0,0)\right)-2(-1)^{q}\left(\cos \left(q k_{x}\right)-1+(\lambda / 2)^{q}\left(\cos \left(q k_{y}\right)-1\right)\right),
$$

the Schrödinger equation becomes

$$
\operatorname{det}\left(m_{p / q}^{(\lambda)}(E, 0,0)\right)=2(-1)^{q}\left(\cos \left(q k_{x}\right)-1+(\lambda / 2)^{q}\left(\cos \left(q k_{y}\right)-1\right)\right) .
$$

Again one introduces the polynomial $b_{p / q}^{(\lambda)}(z)$ and its coefficients $-a_{p / q}^{(\lambda)}(2 j)$ [with $a_{p / q}^{(\lambda)}(0)=-1$,

$$
b_{p / q}^{(\lambda)}(z)=-\sum_{j=0}^{\left[\frac{q}{2}\right]} a_{p / q}^{(\lambda)}(2 j) z^{2 j}
$$

such that $\operatorname{det}\left(m_{p / q}^{(\lambda)}(E, 0,0)\right)$ rewrites as

$$
\operatorname{det}\left(m_{p / q}^{(\lambda)}(E, 0,0)\right)+2(-1)^{q}\left(1+(\lambda / 2)^{q}\right)=(-1)^{q} E^{q} b_{p / q}^{(\lambda)}(1 / E)
$$

so that (24) becomes

$$
E^{q} b_{p / q}^{(\lambda)}(1 / E)=2\left(\cos \left(q k_{x}\right)+(\lambda / 2)^{q} \cos \left(q k_{y}\right)\right) .
$$

In the Appendix, we show how to get a closed expression for the generalized Kreft coefficients $a_{p / q}^{(\lambda)}(2 i)$ 's in (25) following the steps of Ref. 3. This procedure coalesces to

$$
\begin{gathered}
a_{p / q}^{(\lambda)}(2 j)=(-1)^{j+1} \sum_{k_{1}=0}^{q-2 j} \sum_{k_{2}=0}^{k_{1}} \ldots \sum_{k_{j}=0}^{k_{j-1}} \alpha_{p / q}^{(\lambda)}\left(k_{1}+2 j-2\right) \bar{\alpha}_{p / q}^{(\lambda)}\left(k_{1}+2 j-2\right) \\
\alpha_{p / q}^{(\lambda)}\left(k_{2}+2 j-4\right) \bar{\alpha}_{p / q}^{(\lambda)}\left(k_{2}+2 j-4\right) \ldots \alpha_{p / q}^{(\lambda)}\left(k_{j}\right) \bar{\alpha}_{p / q}^{(\lambda)}\left(k_{j}\right)
\end{gathered}
$$

with building blocks

$$
\alpha_{p / q}^{(\lambda)}(k) \bar{\alpha}_{p / q}^{(\lambda)}(k)
$$


where

$$
\alpha_{p / q}^{(\lambda)}(k)=(\lambda / 2) e^{-i k_{y}}\left(1-e^{\frac{2 i \pi(k+1) p}{q}}\right)
$$

and

$$
\bar{\alpha}_{p / q}^{(\lambda)}(k)=(\lambda / 2) e^{i k_{y}}\left(1-(2 / \lambda)^{2} e^{-\frac{2 i \pi(k+1) p}{q}}\right) .
$$

When $\lambda \neq 2$, even though $\bar{\alpha}_{p / q}^{(\lambda)}(k)$ is not anymore the complex conjugate of $\alpha_{p / q}^{(\lambda)}(k)$, it is still true that $a_{p / q}^{(\lambda)}(2 j)$ in (28) is real. Indeed its imaginary part cancels because of

$$
\sum_{k=1}^{q} \mathrm{e}^{\frac{2 \mathrm{i} \pi k p}{q} j}=0
$$

when $q>j$, being a sum of $j$ th powers of $q$ th roots of unity. For example, when $j=1$,

$$
\begin{aligned}
a_{p / q}^{(\lambda)}(2)=\sum_{k=0}^{q-2} \alpha_{p / q}^{(\lambda)}(k) \bar{\alpha}_{p / q}^{(\lambda)}(k) & =(\lambda / 2)^{2} \sum_{k=0}^{q-2}\left(1-e^{\frac{2 i \pi(k+1) p}{q}}+(2 / \lambda)^{2}-(2 / \lambda)^{2} e^{-\frac{2 i \pi(k+1) p}{q}}\right) \\
& =(\lambda / 2)^{2} \sum_{k=0}^{q-1}\left(1-e^{\frac{2 i \pi(k+1) p}{q}}+(2 / \lambda)^{2}-(2 / \lambda)^{2} e^{-\frac{2 i \pi(k+1) p}{q}}\right) \\
& =(\lambda / 2)^{2} \sum_{k=0}^{q-1}\left(1+(2 / \lambda)^{2}\right)=q\left(1+(\lambda / 2)^{2}\right) .
\end{aligned}
$$

Of all that precedes, in the $\lambda \neq 2$ almost Mathieu case, the $\pm s$ spectrum traces and their generating function are directly obtained by replacing in the Hofstadter $\pm s$ spectrum traces and generating functions (19) and (18) the $a_{p / q}(2 j)$ 's by the $a_{p / q}^{(\lambda)}(2 j)$ 's and so the polynomial $b_{p / q}(z)$ by $b_{p / q}^{(\lambda)}(z)$. This is due to the purely algebraic construction of these traces in terms of the $a_{p / q}(2 j)$ 's via the roots of (17) and therefore, in the $\lambda \neq 2$ case, the roots of

$$
E^{q} b_{p / q}^{(\lambda)}(1 / E)= \pm s
$$

with $0 \leq|s| \leq 2\left(1+(\lambda / 2)^{q}\right)$, since from (27) necessarily $0 \leq\left|2\left(\cos \left(q k_{x}\right)+(\lambda / 2)^{q} \cos \left(q k_{y}\right)\right)\right|$ $\leq 2\left(1+(\lambda / 2)^{q}\right)$.

As stated in the Introduction, one wishes to obtain a closed formula for the almost Mathieu quantum trace defined as

$$
\operatorname{Tr}\left(H_{2 \pi p / q}^{(\lambda)}\right)^{n}=\frac{1}{q} \int_{-\pi}^{\pi} \int_{-\pi}^{\pi} \frac{d k_{x}}{2 \pi} \frac{d k_{y}}{2 \pi} \sum_{r=1}^{q}\left(E_{r}^{(\lambda)}\left(k_{x}, k_{y}\right)\right)^{n},
$$

where the $E_{r}^{(\lambda)}\left(k_{x}, k_{y}\right)^{\prime}$ 's are the roots of (27). To do so, as in the Hofstadter case, one aims at integrating over $s \in\left[-2\left(1+(\lambda / 2)^{q}\right), 2\left(1+(\lambda / 2)^{q}\right)\right]$ the $\pm s$ spectrum trace with the density of state $\rho^{(\lambda)}(s)$,

$$
\int_{-2\left(1+(\lambda / 2)^{q}\right)}^{2\left(1+(\lambda / 2)^{q}\right)} \operatorname{Tr}_{s}\left(H_{2 \pi p / q}^{(\lambda)}\right)^{n} \rho^{(\lambda)}(s) d s=\operatorname{Tr}\left(H_{2 \pi p / q}^{(\lambda)}\right)^{n},
$$

which is such that

$$
\int_{-2\left(1+(\lambda / 2)^{q}\right)}^{2\left(1+(\lambda / 2)^{q}\right)} s^{2 k} \rho^{(\lambda)}(s) d s=\left(\begin{array}{c}
2 k \\
k
\end{array}\right) \sum_{k_{1}=0}^{k}\left(\begin{array}{c}
k \\
k_{1}
\end{array}\right)^{2}(\lambda / 2)^{2 q k_{1}}
$$

in analogy to (21). A derivation of $\rho^{(\lambda)}(s)$ enforcing (30) is given in the Appendix. This density of states is the $\lambda$-deformation of $\rho(s)$ in (22) for the $2 \mathrm{~d}$ lattice spectrum $s=2\left(\cos \left(q k_{x}\right)+(\lambda / 2)^{q} \cos \left(q k_{y}\right)\right)$.

Putting all the steps above together, namely, in (19) replacing both the $a_{p / q}(2 j)$ 's by the $a_{p / q}^{(\lambda)}(2 j)$ 's and $s^{2 k}$ by $\left(\begin{array}{c}2 k \\ k\end{array}\right) \sum_{k_{1}=0}^{k}\left(\begin{array}{c}k \\ k_{1}\end{array}\right)^{2}(\lambda / 2)^{2 q k_{1}}$, the almost Mathieu operator quantum trace (29) ends up as 


$$
\begin{aligned}
\operatorname{Tr}\left(H_{2 \pi p / q}^{(\lambda)}\right)^{n}= & \frac{n}{q} \sum_{k \geq 0} \sum_{\substack{\ell_{1}, \ell_{2}, \ldots, \ell_{\lfloor q / 2\rfloor} \geq 0 \\
\ell_{1}+2 \ell_{2}+\cdots+\lfloor q / 2\rfloor \ell_{\lfloor q / 2\rfloor}=n / 2-q k}} \frac{\left(\begin{array}{c}
\ell_{1}+\ell_{2}+\cdots+\ell_{\lfloor q / 2\rfloor}+2 k \\
\ell_{1}, \ell_{2}, \ldots, \ell_{\lfloor q / 2\rfloor}, 2 k
\end{array}\right)}{\ell_{1}+\ell_{2}+\cdots+\ell_{\lfloor q / 2\rfloor}+2 k}\left(\begin{array}{c}
2 k \\
k
\end{array}\right) \sum_{k_{1}=0}^{k}\left(\begin{array}{c}
k \\
k_{1}
\end{array}\right)^{2}(\lambda / 2)^{2 q k_{1}} \\
& \prod_{j=1}^{\lfloor q / 2\rfloor} a_{p / q}^{(\lambda)}(2 j)^{\ell_{j}},
\end{aligned}
$$

which is a $\lambda$-deformation of the quantum Hofstadter trace (11).

One also gets the generating function that generalizes (12),

$$
\sum_{n \geq 0} Z_{n}^{(\lambda)}\left(e^{2 i \pi p / q}\right) z^{n}=\left(1-\frac{z\left(b_{p / q}^{(\lambda)}(z)\right)^{\prime}}{q b_{p / q}^{(\lambda)}(z)}\right) \sum_{k \geq 0}\left(\begin{array}{c}
2 k \\
k
\end{array}\right) \sum_{k_{1}=0}^{k}\left(\begin{array}{c}
k \\
k_{1}
\end{array}\right)^{2}(\lambda / 2)^{2 q k_{1}}\left(\frac{z^{q}}{b_{p / q}^{(\lambda)}(z)}\right)^{2 k},
$$

where $\sum_{k \geq 0}\left(\begin{array}{c}2 k \\ k\end{array}\right) \sum_{k_{1}=0}^{k}\left(\begin{array}{l}k \\ k_{1}\end{array}\right)^{2}(\lambda / 2)^{2 q k_{1}} x^{k}$ can be viewed again in terms of the complete elliptic integral $(2 / \pi) K(16 x)=\sum_{k \geq 0}\left(\begin{array}{c}2 k \\ k\end{array}\right)^{2} x^{k}$. Indeed thanks to the identity

$$
\sum_{k=0}^{\infty}\left(\begin{array}{c}
2 k \\
k
\end{array}\right) x^{k} \sum_{k_{1}=0}^{k}\left(\begin{array}{c}
k \\
k_{1}
\end{array}\right)^{2} y^{2 k_{1}}=\frac{1}{\sqrt{1-4 x(1-y)^{2}}} \sum_{k=0}^{\infty}\left(\begin{array}{c}
2 k \\
k
\end{array}\right)^{2}\left(\frac{x y}{1-4 x(1-y)^{2}}\right)^{k},
$$

(32) can be summed up to

$$
\begin{aligned}
\sum_{n \geq 0} Z_{n}^{(\lambda)}\left(e^{2 i \pi p / q}\right) z^{n}= & \left(1-\frac{z\left(b_{p / q}^{(\lambda)}(z)\right)^{\prime}}{q b_{p / q}^{(\lambda)}(z)}\right) \frac{1}{\sqrt{1-4\left(\frac{z^{q}}{b_{p / q}^{(\lambda)}(z)}\right)^{2}\left(1-(\lambda / 2)^{q}\right)^{2}}} \\
& \times \frac{2}{\pi} K\left(\frac{16 z^{2 q}(\lambda / 2)^{q}}{b_{p / q}^{(\lambda)}(z)^{2}-4 z^{2 q}\left(1-(\lambda / 2)^{q}\right)^{2}}\right)
\end{aligned}
$$

which rightly reduces to (12) when $\lambda=2$ [see Subsection 2 in the Appendix for a derivation of the density of state $\rho^{(\lambda)}(s)$ again in terms of the complete elliptic integral]. One can also check that the expression (34) for the generating function can be retrieved by starting from (29) and integrating with respect to the momenta $k_{x}$ and $k_{y}$.

To see why (33) holds, first note that $\sum_{k_{1}=0}^{k}\left(\begin{array}{l}k \\ k_{1}\end{array}\right)^{2} y^{2 k_{1}}$ is the coefficient of $\boldsymbol{t}^{\mathbf{0}}$ in

$$
\left(1+\frac{y}{t}\right)^{k}(1+y t)^{k}=\sum_{i=0}^{k}\left(\begin{array}{l}
k \\
i
\end{array}\right) y^{i} t^{-i} \sum_{j=0}^{k}\left(\begin{array}{l}
k \\
j
\end{array}\right) y^{j} t^{j} .
$$

Thus the left-hand side of (33) is the coefficient of $t^{0}$ in

$$
\begin{aligned}
\sum_{k=0}^{\infty}\left(\begin{array}{c}
2 k \\
k
\end{array}\right) x^{k}\left(1+\frac{y}{t}\right)^{k}(1+y t)^{k} & =\frac{1}{\sqrt{1-4 x(1+y / t)(1+y t)}} \\
& =\frac{1}{\sqrt{1-4 x(1-y)^{2}-4 x y(1+t)^{2} / t}} \\
& =\frac{1}{\sqrt{1-4 x(1-y)^{2}}} \cdot \frac{1}{\sqrt{1-4 \frac{x y}{1-4 x(1-y)^{2}} \cdot \frac{(1+t)^{2}}{t}}} \\
& =\frac{1}{\sqrt{1-4 x(1-y)^{2}}} \cdot \sum_{k=0}^{\infty}\left(\begin{array}{c}
2 k \\
k
\end{array}\right)\left(\frac{x y}{1-4 x(1-y)^{2}}\right)^{k}\left(\frac{(1+t)^{2}}{t}\right)^{k} .
\end{aligned}
$$


Now it only remains to see that the coefficient of $\boldsymbol{t}^{\mathbf{0}}$ in $\left(\frac{(1+t)^{2}}{t}\right)^{k}$ is precisely $\left(\begin{array}{c}2 k \\ k\end{array}\right)$.

Note finally that one can check that the Aubry duality, ${ }^{9}$

$$
\operatorname{Tr}\left(H_{2 \pi p / q}^{(\lambda)}\right)^{n}=\left(\frac{\lambda}{2}\right)^{n} \operatorname{Tr}\left(H_{2 \pi p / q}^{(4 / \lambda)}\right)^{n}
$$

holds, as it should. This happens because the generalized Kreft coefficients (28) themselves obey the duality

$$
a_{p / q}^{(\lambda)}(2 j)=\left(\frac{\lambda}{2}\right)^{2 j} a_{p / q}^{(4 / \lambda)}(2 j) \Rightarrow b_{p / q}^{(\lambda)}(z)=b_{p / q}^{(4 / \lambda)}\left(\frac{\lambda z}{2}\right)
$$

which follows from

$$
\operatorname{Re}\left(\alpha_{p / q}^{(\lambda)}(k) \bar{\alpha}_{p / q}^{(\lambda)}(k)\right)=\left(\frac{\lambda}{2}\right)^{2} \operatorname{Re}\left(\alpha_{p / q}^{(4 / \lambda)}(k) \bar{\alpha}_{p / q}^{(4 / \lambda)}(k)\right)
$$

[only the real part is needed here since $a_{p / q}^{(\lambda)}(2 j)$ is real]. The duality (35) then follows from (31) and the duality (36),

$$
\begin{aligned}
& \operatorname{Tr}\left(H_{2 \pi p / q}^{(\lambda)}\right)^{n}=\frac{n}{q} \sum_{k \geq 0} \sum_{\substack{\ell_{1}, \ell_{2}, \ldots, \ell_{\lfloor q / 2\rfloor} \geq 0 \\
\ell_{1}+2 \ell_{2}+\cdots+\lfloor q / 2\rfloor \ell_{\lfloor q / 2\rfloor}=n / 2-q k}} \frac{\left(\begin{array}{c}
2 k \\
k
\end{array}\right) \sum_{k_{1}=0}^{k}\left(\begin{array}{c}
k \\
k_{1}
\end{array}\right)^{2}(\lambda / 2)^{2 q k_{1}}\left(\begin{array}{c}
\ell_{1}+\ell_{2}+\cdots+\ell_{\lfloor q / 2\rfloor}+2 k \\
\ell_{1}, \ell_{2}, \ldots, \ell_{\lfloor q / 2\rfloor}, 2 k
\end{array}\right)}{\ell_{1}+\ell_{2}+\cdots+\ell_{\lfloor q / 2\rfloor}+2 k} \\
& (\lambda / 2)^{\sum_{j=1}^{\lfloor q / 2\rfloor} 2 j l_{j}} \prod_{j=1}^{\lfloor q / 2\rfloor} a_{p / q}^{(4 / \lambda)}(2 j)^{\ell_{j}} \\
& =\frac{n}{q} \sum_{k \geq 0} \sum_{\substack{\ell_{1}, \ell_{2}, \ldots, \ell_{\lfloor q / 2\rfloor} \geq 0 \\
\ell_{1}+2 \ell_{2}+\cdots+\lfloor q / 2\rfloor \ell_{\lfloor q / 2\rfloor}=n / 2-q k}} \frac{\left(\begin{array}{c}
2 k \\
k
\end{array}\right) \sum_{k_{1}=0}^{k}\left(\begin{array}{c}
k \\
k_{1}
\end{array}\right)^{2}(\lambda / 2)^{2 q k_{1}}\left(\begin{array}{c}
\ell_{1}+\ell_{2}+\cdots+\ell_{\lfloor q / 2\rfloor}+2 k \\
\ell_{1}, \ell_{2}, \ldots, \ell_{\lfloor q / 2\rfloor}, 2 k
\end{array}\right)}{\ell_{1}+\ell_{2}+\cdots+\ell_{\lfloor q / 2\rfloor}+2 k} \\
& (\lambda / 2)^{n-2 q k} \prod_{j=1}^{\lfloor q / 2\rfloor} a_{p / q}^{(4 / \lambda)}(2 j)^{\ell_{j}} \\
& =(\lambda / 2)^{n} \frac{n}{q} \sum_{k \geq 0} \sum_{\begin{array}{c}
\ell_{1}, \ell_{2}, \ldots, \ell_{\lfloor q / 2\rfloor} \geq 0 \\
\ell_{1}+2 \ell_{2}+\cdots+\lfloor q / 2\rfloor \ell_{\lfloor q / 2\rfloor}=n / 2-q k
\end{array}} \frac{\left(\begin{array}{c}
2 k \\
k
\end{array}\right) \sum_{k_{1}=0}^{k}\left(\begin{array}{c}
k \\
k_{1}
\end{array}\right)^{2}(2 / \lambda)^{2 q k-2 q k_{1}\left(\begin{array}{c}
\ell_{1}+\ell_{2}+\cdots+\ell_{[q / 2\rfloor}+2 k \\
\ell_{1}, \ell_{2}, \ldots, \ell_{\lfloor q / 2\rfloor}, 2 k
\end{array}\right)}}{\ell_{1}+\ell_{2}+\cdots+\ell_{\lfloor q / 2\rfloor}+2 k} \\
& \prod_{j=1}^{\lfloor q / 2\rfloor} a_{p / q}^{(4 / \lambda)}(2 j)^{\ell_{j}} \\
& \stackrel{k_{1}^{\prime}=k-k_{1}}{=}(\lambda / 2)^{n} \frac{n}{q} \sum_{k \geq 0} \sum_{\substack{\ell_{1}, \ell_{2}, \ldots, \ell_{\lfloor q / 2\rfloor} \geq 0 \\
\ell_{1}+2 \ell_{2}+\cdots+\lfloor q / 2\rfloor \ell_{\lfloor q / 2\rfloor}=n / 2-q k}} \frac{\left(\begin{array}{c}
2 k \\
k
\end{array}\right) \sum_{k_{1}^{\prime}=0}^{k}\left(\begin{array}{c}
k \\
k_{1}^{\prime}
\end{array}\right)^{2}(2 / \lambda)^{2 q k_{1}^{\prime}\left(\begin{array}{c}
\ell_{1}+\ell_{2}+\cdots+\ell_{\lfloor q / 2\rfloor}+2 k \\
\ell_{1}, \ell_{2}, \ldots, \ell_{\lfloor q / 2\rfloor}, 2 k
\end{array}\right)}}{\ell_{1}+\ell_{2}+\cdots+\ell_{\lfloor q / 2\rfloor}+2 k} \\
& \prod_{j=1}^{\lfloor q / 2\rfloor} a_{p / q}^{(4 / \lambda)}(2 j)^{\ell_{j}}=(\lambda / 2)^{n} \operatorname{Tr}\left(H_{2 \pi p / q}^{(4 / \lambda)}\right)^{n} .
\end{aligned}
$$

\section{CONCLUSION}

One has obtained the $\lambda$-deformation of the quantum trace (11) in the form of (31). Both trace formulae have a similar structure, with clearly (31), the almost Mathieu case, reducing when $\lambda=2$ to (11), the Hofstadter case. Going back for a moment to random walks on a lattice, it would certainly be interesting to look at possible interpretations of (31) in the context of asymmetric paths with unequal probabilities on the horizontal and vertical axis.

On the other hand, our results are directly relevant for problems related to Calabi-Yau geometry. A recent work ${ }^{10}$ indicates that there exists in the rational case a relation between the almost Mathieu 
operator and the relativistic Toda lattice. As pointed out in Sec. 2 of Ref. 10, there is an invariance under the modular double operation exchanging $\gamma=2 \pi p / q$ and $\tilde{\gamma}=2 \pi q / p$ (respectively, $\hbar$ and $\tilde{\hbar}$ in the notations of Ref. 10) of the relativistic relative 2-body Toda Hamiltonian

$$
H=\lambda\left(e^{p}+e^{-p}\right)+e^{x}+e^{-x}, \quad[x, p]=i \gamma,
$$

where, in Ref. 10, $\lambda$ is denoted as $R^{2}$. The eigenvalues $E$ and $\tilde{E}$ corresponding to $\gamma$ and $\tilde{\gamma}$ satisfy the polynomial identity $P_{p / q}(E)=P_{q / p}(\tilde{E})$, where $P_{p / q}(E)$ is a polynomial of degree $q$ [see (2.19) in Ref. 10]. Now this polynomial is identical to the polynomial $E^{q} b_{p / q}^{(\lambda)}(1 / E)$ introduced in (25) and (26) which encodes the $\left\{k_{x}, k_{y}\right\}$-independent part of the determinant of the almost Mathieu Schrödinger equation encapsulated in $m_{p / q}^{(\lambda)}\left(E, k_{x}, k_{y}\right)$. In the present work, we have precisely obtained in (28) a closed expression for these polynomials in terms of the generalized Kreft coefficients.

It would certainly be rewarding to see if (28) can bring any pertinent information related to the various questions raised in Ref. 10, in particular, regarding the quantum A-period for the modulus of the underlying Calabi-Yau geometry. Finally, in the context of the Hofstadter model itself, the true role of the double modular transformation $\gamma \rightarrow \tilde{\gamma}$ remains to be elucidated.

\section{ACKNOWLEDGMENTS}

S.W. was supported by the National Research Foundation of South Africa, Grant No. 96236. S.O. would like to thank S. Nechaev for drawing his attention to Ref. 10.

\section{APPENDIX A: KREFT'S COEFFICIENTS CONSTRUCTION}

Following Kreft, ${ }^{3}$ we show how to get a closed expression for the polynomial (26), i.e., for the Kreft coefficients $a_{p / q}^{(\lambda)}(2 j)$ in $b_{p / q}^{(\lambda)}(z)=-\sum_{j=0}^{\left[\frac{q}{2}\right]} a_{p / q}^{(\lambda)}(2 j) z^{2 j}$ as defined in (25).

1. $\lambda=2$

One aims at transforming the matrix $m_{p / q}\left(E, k_{x}, k_{y}\right)$ in (3) into a tridiagonal one by an appropriate change of basis. First, let us do the change of basis

$$
m_{1}=\left(\begin{array}{cccccc}
1 & 0 & 0 & \cdots & 0 & 0 \\
0 & e^{i k_{x}} & 0 & \cdots & 0 & 0 \\
0 & 0 & e^{i 2 k_{x}} & \cdots & 0 & 0 \\
\vdots & \vdots & \vdots & \ddots & \vdots & \vdots \\
0 & 0 & 0 & \cdots & e^{i(q-2) k_{x}} & 0 \\
0 & 0 & 0 & \cdots & 0 & e^{i(q-1) k_{x}}
\end{array}\right)
$$

so that $m_{p / q}\left(E, k_{x}, k_{y}\right)$ rewrites as

$$
\begin{aligned}
& m_{1}^{-1} m_{p / q}\left(E, k_{x}, k_{y}\right) m_{1} \\
& =\left(\begin{array}{cccccc}
2 \cos \left(k_{y}\right)-E & e^{i k_{x}} & 0 & \cdots & 0 & e^{-i k_{x}} \\
e^{-i k_{x}} & 2 \cos \left(k_{y}+\frac{2 \pi p}{q}\right)-E & e^{i k_{x}} & \cdots & 0 & 0 \\
0 & e^{-i k_{x}} & () & \cdots & 0 & 0 \\
\vdots & \vdots & \vdots & \ddots & \vdots & \vdots \\
0 & 0 & 0 & \cdots & () & e^{i k_{x}} \\
e^{i k_{x}} & 0 & 0 & \cdots & e^{-i k_{x}} & 2 \cos \left(k_{y}+(q-1) \frac{2 \pi p}{q}\right)-E
\end{array}\right) .
\end{aligned}
$$

In this new basis, the Schrödinger equation (1) with $\Phi_{m+q}=e^{i q k_{x}} \Phi_{m}$ becomes

$$
e^{i k_{x}} \Phi_{m+1}^{\prime}+e^{-i k_{x}} \Phi_{m-1}^{\prime}+2 \cos \left(k_{y}+\gamma m\right) \Phi_{m}^{\prime}=E \Phi_{m}^{\prime}, \quad \Phi_{m+q}^{\prime}=\Phi_{m}^{\prime} .
$$


Then let us do a second change of basis $m_{2}$ with matrix element

$$
m_{j k}=\frac{1}{\sqrt{q}}(-1)^{p k} e^{\frac{2 i \pi p}{q}\left(j k-k^{2} / 2\right)}, \quad k, j \in\{0,1, \ldots, q-1\} .
$$

Putting together $m_{1}$ and $m_{2}$ amounts to the change of basis $m_{1} m_{2}$ with matrix element $e^{i k k_{x}} m_{j k}=$ $e^{i k k_{x}} \frac{1}{\sqrt{q}}(-1)^{p k} e^{\frac{2 i \pi p}{q}\left(j k-k^{2} / 2\right)}$,

$$
\left(m_{1} m_{2}\right)^{-1} m_{p / q}\left(E, k_{x}, k_{y}\right) m_{1} m_{2}=\left(\begin{array}{cccccc}
-E & \alpha_{p / q}(0) & 0 & \cdots & 0 & \bar{\alpha}_{p / q}(q-1) \\
\bar{\alpha}_{p / q}(0) & -E & \alpha_{p / q}(1) & \cdots & 0 & 0 \\
0 & \bar{\alpha}_{p / q}(1) & -E & \cdots & 0 & 0 \\
\vdots & \vdots & \vdots & \ddots & \vdots & \vdots \\
0 & 0 & 0 & \cdots & -E & \alpha_{p / q}(q-2) \\
\alpha_{p / q}(q-1) & 0 & 0 & \cdots & \alpha_{p / q}(q-2) & -E
\end{array}\right) \text {, }
$$

where $\alpha_{p / q}(k)=e^{-i k_{y}}-(-1)^{(p+1)} e^{\frac{2 i \pi p}{q}(k+1 / 2)+i k_{x}}$ and $\bar{\alpha}_{p / q}(k)$ is its complex conjugate and accordingly

$$
\alpha_{p / q}(m) \tilde{\Phi}_{m+1}+\bar{\alpha}_{p / q}(m-1) \tilde{\Phi}_{m-1}=E \tilde{\Phi}_{m}, \quad \tilde{\Phi}_{m+q}=\tilde{\Phi}_{m} .
$$

Both corners $\alpha_{p / q}(q-1)$ and $\bar{\alpha}_{p / q}(q-1)$ in the matrix (A1) can be canceled if $e^{i\left(k_{x}+k_{y}\right)}=$ $(-1)^{(p+1)} e^{-\frac{2 i \pi p}{q}(q-1+1 / 2)}=(-1)^{(p+1)} e^{\frac{i \pi p}{q}}$, i.e., if $k_{x}+k_{y}=\pi(p+1)+\frac{\pi p}{q}$. The matrix is then tridiagonal with a determinant in (4) equal to $(-1)^{q} E^{q} b_{p / q}(1 / E)$, since the trigonometric part vanishes as well; indeed

$$
\begin{aligned}
\cos \left(q k_{x}\right)+\cos \left(q k_{y}\right) & =2 \cos \left(\frac{q\left(k_{x}+k_{y}\right)}{2}\right) \cos \left(\frac{q\left(k_{x}-k_{y}\right)}{2}\right) \\
& =2 \cos \left(\frac{\pi(p+q(p+1))}{2}\right) \cos \left(\frac{q\left(k_{x}-k_{y}\right)}{2}\right) \\
& =0
\end{aligned}
$$

due to $p+q(p+1)$ being always odd since $p$ and $q$ are co-prime.

One gets the tridiagonal matrix

$$
\left(\begin{array}{cccccc}
-E & \alpha_{p / q}(0) & 0 & \cdots & 0 & 0 \\
\bar{\alpha}_{p / q}(0) & -E & \alpha_{p / q}(1) & \cdots & 0 & 0 \\
0 & \bar{\alpha}_{p / q}(1) & -E & \cdots & 0 & 0 \\
\vdots & \vdots & \vdots & \ddots & \vdots & \vdots \\
0 & 0 & 0 & \cdots & -E & \alpha_{p / q}(q-2) \\
0 & 0 & 0 & \cdots & \bar{\alpha}_{p / q}(q-2) & -E
\end{array}\right),
$$


where $\alpha_{p / q}(k)$, using $e^{i\left(k_{x}+k_{y}\right)}=(-1)^{(p+1)} e^{\frac{i \pi p}{q}}$, has simplified to $\alpha_{p / q}(k)=e^{-i k_{y}}\left(1-e^{\frac{2 i \pi(k+1) p}{q}}\right)$. The matrix (A3) does not depend anymore on $k_{x}$ nor on $k_{y}$ and its determinant satisfies a recursion (see Ref. 3) leading, respectively, to the Kreft polynomial and Kreft coefficients (7) and (9).

\section{2. $\lambda \neq 2$}

One uses the same method as above to find a closed expression for the polynomial $b_{p / q}^{(\lambda)}(1 / E)$, namely, transform the matrix $m_{p / q}^{(\lambda)}\left(E, k_{x}, k_{y}\right)$ in (23) into a tridiagonal one. To do so, use the same change of basis $m_{1} m_{2}$ as above so that

$$
\begin{aligned}
& \left(m_{1} m_{2}\right)^{-1} m_{p / q}^{(\lambda)}\left(E, k_{x}, k_{y},\right) m_{1} m_{2} \\
& \quad=\left(\begin{array}{cccccc}
-E & \alpha_{p / q}^{(\lambda)}(0) & 0 & \ldots & 0 & \bar{\alpha}_{p / q}^{(\lambda)}(q-1) \\
\bar{\alpha}_{p / q}^{(\lambda)}(0) & -E & \alpha_{p / q}^{(\lambda)}(1) & \ldots & 0 & 0 \\
0 & \bar{\alpha}_{p / q}^{(\lambda)}(1) & -E & \ldots & 0 & 0 \\
\vdots & \vdots & \vdots & \ddots & \vdots & \vdots \\
0 & 0 & 0 & \ldots & -E & \alpha_{p / q}^{(\lambda)}(q-2) \\
\alpha_{p / q}^{(\lambda)}(q-1) & 0 & 0 & \ldots & \bar{\alpha}_{p / q}^{(\lambda)}(q-2) & -E
\end{array}\right)
\end{aligned}
$$

with $\alpha_{p / q}^{(\lambda)}(k)=(\lambda / 2) e^{-i k_{y}}-(-1)^{p+1} e^{i \frac{2 \pi p}{q}(k+1 / 2)+i k_{x}}$ and $\bar{\alpha}_{p / q}^{(\lambda)}(k)$ its complex conjugate. Moreover, we have a resulting Schrödinger equation identical to (A2) provided that $\alpha_{p / q}(k)$ is replaced with $\alpha_{p / q}^{(\lambda)}(k)$.

Contrary to the Hofstadter case $\lambda=2$, both corners $\alpha_{p / q}^{(\lambda)}(q-1)$ and $\bar{\alpha}_{p / q}^{(\lambda)}(q-1)$ in the matrix (A4) cannot simultaneously vanish. One can still choose to have the lower left corner $\alpha_{p / q}^{(\lambda)}(q-1)$ to vanish: this amounts to $e^{i\left(k_{x}+k_{y}\right)}=(-1)^{p+1}(\lambda / 2) e^{-i \frac{2 \pi p}{q}(q-1+1 / 2)}=(-1)^{p+1}(\lambda / 2) e^{\frac{i \pi p}{q}}$, which can only be achieved for a complex $k_{x}+k_{y}$ [namely, $\left.k_{x}+k_{y}=-i \log (\lambda / 2)+\frac{\pi p}{q}+\pi(p+1)\right]$ so that $e^{-i\left(k_{x}+k_{y}\right)}=(-1)^{p+1}(2 / \lambda) e^{-\frac{i \pi p}{q}}$. The matrix (A4) then becomes

$$
\left(\begin{array}{cccccc}
-E & \alpha_{p / q}^{(\lambda)}(0) & 0 & \cdots & 0 & \bar{\alpha}_{p / q}^{(\lambda)}(q-1) \\
\bar{\alpha}_{p / q}^{(\lambda)}(0) & -E & \alpha_{p / q}^{(\lambda)}(1) & \cdots & 0 & 0 \\
0 & \bar{\alpha}_{p / q}^{(\lambda)}(1) & -E & \cdots & 0 & 0 \\
\vdots & \vdots & \vdots & \ddots & \vdots & \vdots \\
0 & 0 & 0 & \cdots & -E & \alpha_{p / q}^{(\lambda)}(q-2) \\
0 & 0 & 0 & \cdots & \bar{\alpha}_{p / q}^{(\lambda)}(q-2) & -E
\end{array}\right)
$$

where $\alpha_{p / q}^{(\lambda)}(k)$ and $\bar{\alpha}_{p / q}^{(\lambda)}(k)$ have simplified to $\alpha_{p / q}^{(\lambda)}(k)=(\lambda / 2) e^{-i k_{y}}\left(1-e^{\frac{2 i \pi(k+1) p}{q}}\right)$ and $\bar{\alpha}_{p / q}^{(\lambda)}(k)$ $=(\lambda / 2) e^{i k_{y}}\left(1-(2 / \lambda)^{2} e^{-\frac{2 i \pi(k+1) p}{q}}\right)$. Note that $\bar{\alpha}_{p / q}^{(\lambda)}(k)$ is not anymore the complex conjugate of $\alpha_{p / q}^{(\lambda)}(k)$.

By expanding the determinant of (A5) with respect to the elements of the first row 


$$
\begin{aligned}
& \left|\begin{array}{cccccc}
-E & \alpha_{p / q}^{(\lambda)}(0) & 0 & \ldots & 0 & \bar{\alpha}_{p / q}^{(\lambda)}(q-1) \\
\bar{\alpha}_{p / q}^{(\lambda)}(0) & -E & \alpha_{p / q}^{(\lambda)}(1) & \ldots & 0 & 0 \\
0 & \bar{\alpha}_{p / q}^{(\lambda)}(1) & -E & \ldots & 0 & 0 \\
\vdots & \vdots & \vdots & \ddots & \vdots & \vdots \\
0 & 0 & 0 & \ldots & -E & \alpha_{p / q}^{(\lambda)}(q-2) \\
0 & 0 & 0 & \ldots & \bar{\alpha}_{p / q}^{(\lambda)}(q-2) & -E
\end{array}\right|=-E\left|\begin{array}{ccccc}
-E & \alpha_{p / q}^{(\lambda)}(1) & \ldots & 0 & 0 \\
\bar{\alpha}_{p / q}^{(\lambda)}(1) & -E & \ldots & 0 & 0 \\
\vdots & \vdots & \ddots & \vdots & \vdots \\
0 & 0 & \ldots & -E & \alpha_{p / q}^{(\lambda)}(q-2) \\
0 & 0 & \ldots & \bar{\alpha}_{p / q}^{(\lambda)}(q-2) & -E
\end{array}\right| \\
& -\alpha_{p / q}^{(\lambda)}(0) \overline{\boldsymbol{\alpha}}_{p / q}^{(\lambda)}\left|\begin{array}{ccccc}
-E & \alpha_{p / q}^{(\lambda)}(2) & \cdots & 0 & 0 \\
\overline{\boldsymbol{\alpha}}_{p / q}^{(\lambda)}(2) & -E & \cdots & 0 & 0 \\
\vdots & \vdots & \ddots & \vdots & \vdots \\
0 & 0 & \cdots & -E & \alpha_{p / q}^{(\lambda)}(q-2) \\
0 & 0 & \cdots & \overline{\boldsymbol{\alpha}}_{p / q}^{(\lambda)}(q-2) & -E
\end{array}\right| \\
& +(-1)^{q+1} \overline{\boldsymbol{\alpha}}_{p / q}^{(\lambda)}(q-1)\left|\begin{array}{cccccc}
\bar{\alpha}_{p / q}^{(\lambda)}(0) & -E & \alpha_{p / q}^{(\lambda)}(1) & \ldots & 0 & 0 \\
0 & \overline{\boldsymbol{\alpha}}_{p / q}^{(\lambda)}(1) & -E & \ldots & 0 & 0 \\
\vdots & \vdots & \vdots & \ddots & \vdots & \vdots \\
0 & 0 & 0 & \ldots & \overline{\boldsymbol{\alpha}}_{p / q}^{(\lambda)}(q-3) & -E \\
0 & 0 & 0 & \ldots & 0 & \bar{\alpha}_{p / q}^{(\lambda)}(q-2)
\end{array}\right|,
\end{aligned}
$$

it is immediate to see that the part that depends on $k_{x}$ or $k_{y}$ can only come from the last term of (A6), i.e., from the upper right corner $\bar{\alpha}_{p / q}^{(\lambda)}(q-1)$. Therefore to get the desired $k_{x}, k_{y}$-independent polynomial $E^{q} b_{p / q}^{(\lambda)}(1 / E)$, all that is needed is the determinant of the tridiagonal matrix

$$
\left(\begin{array}{cccccc}
-E & \alpha_{p / q}^{(\lambda)}(0) & 0 & \cdots & 0 & 0 \\
\bar{\alpha}_{p / q}^{(\lambda)}(0) & -E & \alpha_{p / q}^{(\lambda)}(1) & \ldots & 0 & 0 \\
0 & \bar{\alpha}_{p / q}^{(\lambda)}(1) & -E & \ldots & 0 & 0 \\
\vdots & \vdots & \vdots & \ddots & \vdots & \vdots \\
0 & 0 & 0 & \ldots & -E & \alpha_{p / q}^{(\lambda)}(q-2) \\
0 & 0 & 0 & \ldots & \bar{\alpha}_{p / q}^{(\lambda)}(q-2) & -E
\end{array}\right)
$$

which finally yields the $\lambda \neq 2$ Kreft polynomial and Kreft coefficients (26) and (28).

\section{APPENDIX B: DENSITY OF STATES $\rho^{(\lambda)}(s)$}

To simplify the notations, let us denote in this section $(\lambda / 2)^{q}$ by $\tilde{\lambda}$. Knowing that

$$
\int_{-4}^{4} \rho(s) s^{2 k} d s=\left(\begin{array}{c}
2 k \\
k
\end{array}\right)^{2}
$$

for $\rho(s)=\left(2 \pi^{2}\right)^{-1} K\left(1-s^{2} / 16\right)$, where $K$ denotes the elliptic integral, we would like to determine a function $\rho^{(\lambda)}(s)$ such that

$$
\int_{-2(1+\tilde{\lambda})}^{2(1+\tilde{\lambda})} \rho^{(\lambda)}(s) s^{2 k} d s=\left(\begin{array}{c}
2 k \\
k
\end{array}\right) \sum_{k_{1}=0}^{k}\left(\begin{array}{c}
k \\
k_{1}
\end{array}\right)^{2} \tilde{\lambda}^{2 k_{1}}
$$

as in (30). The special case $\tilde{\lambda}=1$ clearly corresponds to the aforementioned formula. 
We interpret the desired function $\rho^{(\lambda)}(s)$ as the density of a random variable with support $[-2(1+$ $\tilde{\lambda}), 2(1+\tilde{\lambda})]$ that is symmetric (so that the odd-order moments are 0 ) and has $2 k$ th moment

$$
M_{2 k}=\left(\begin{array}{c}
2 k \\
k
\end{array}\right) \sum_{k_{1}=0}^{k}\left(\begin{array}{c}
k \\
k_{1}
\end{array}\right)^{2} \tilde{\lambda}^{2 k_{1}} .
$$

The moment generating function associated with this random variable is

$$
\begin{aligned}
\sum_{k=0}^{\infty} \frac{M_{2 k}}{(2 k) !} x^{2 k} & =\sum_{k=0}^{\infty}\left(\begin{array}{c}
2 k \\
k
\end{array}\right) \sum_{k_{1}=0}^{k}\left(\begin{array}{c}
k \\
k_{1}
\end{array}\right)^{2} \tilde{\lambda}^{2 k_{1}} \frac{x^{2 k}}{(2 k) !} \\
& =\sum_{k_{1}=0}^{\infty} \sum_{k=k_{1}}^{\infty}\left(\begin{array}{c}
2 k \\
k
\end{array}\right)\left(\begin{array}{l}
k \\
k_{1}
\end{array}\right)^{2} \tilde{\lambda}^{2 k_{1}} \frac{x^{2 k}}{(2 k) !} \\
& =\sum_{k_{1}=0}^{\infty} \frac{\tilde{\lambda}^{2 k_{1}}}{k_{1} !^{2}} \sum_{k=k_{1}}^{\infty} \frac{1}{\left(k-k_{1}\right) !^{2}} x^{2 k} \\
& =\sum_{k_{1}=0}^{\infty} \frac{\tilde{\lambda}^{2 k_{1}}}{k_{1} !^{2}} \sum_{j=0}^{\infty} \frac{1}{j !^{2}} x^{2\left(k_{1}+j\right)} \\
& =\sum_{k_{1}=0}^{\infty} \frac{\tilde{\lambda}^{2 k_{1}} x^{2 k_{1}}}{k_{1} !^{2}} \sum_{j=0}^{\infty} \frac{x^{2 j}}{j !^{2}} \\
& =I(2 \tilde{\lambda} x) I(2 x),
\end{aligned}
$$

where $I$ denotes the 0 -th order modified Bessel function of the first kind. Thus the random variable whose density $\rho^{(\lambda)}(s)$ we would like to determine is the convolution of two random variables with moment generating functions $I(2 \tilde{\lambda} x)$ and $I(2 x)$, respectively.

Now note that $I(2 x)$ is exactly the moment generating function of an arcsine distribution on the interval $[-2,2]$,

$$
\int_{-2}^{2} \frac{e^{s x}}{\pi \sqrt{4-s^{2}}} d s=I(2 x)
$$

and likewise $I(2 \tilde{\lambda} x)$ is the moment generating function of an arcsine distribution on the interval $[-2 \tilde{\lambda}, 2 \tilde{\lambda}]$,

$$
\int_{-2 \tilde{\lambda}}^{2 \tilde{\lambda}} \frac{e^{s x}}{\pi \sqrt{4 \tilde{\lambda}^{2}-s^{2}}} d s=I(2 \tilde{\lambda} x) .
$$

Therefore $\rho^{(\lambda)}(s)$ must be the convolution of the two densities $h_{1}(s)=\left(\pi \sqrt{4-s^{2}}\right)^{-1}(s \in[-2,2]$, otherwise $\left.h_{1}(s)=0\right)$ and $h_{2}(s)=\left(\pi \sqrt{4 \tilde{\lambda}^{2}-s^{2}}\right)^{-1}\left[s \in[-2 \tilde{\lambda}, 2 \tilde{\lambda}]\right.$, otherwise $\left.h_{2}(s)=0\right]$, which is

$$
\rho^{(\lambda)}(s)=\int_{-\infty}^{\infty} h_{1}(s-t) h_{2}(t) d t
$$

This reduces to several different cases:

- Case 1: $\tilde{\lambda}>1$

(1) if $s<-2-2 \tilde{\lambda}$ or $s>2+2 \tilde{\lambda}$, then $\rho^{(\lambda)}(s)=0$,

(2) if $-2-2 \tilde{\lambda} \leq s<2-2 \tilde{\lambda}$, then

$$
\rho^{(\lambda)}(s)=\pi^{-2} \int_{-2 \tilde{\lambda}}^{s+2} \frac{1}{\sqrt{\left(4-(s-t)^{2}\right)\left(4 \tilde{\lambda}^{2}-t^{2}\right)}} d t,
$$

(3) if $2-2 \tilde{\lambda} \leq s \leq 2 \tilde{\lambda}-2$, then

$$
\rho^{(\lambda)}(s)=\pi^{-2} \int_{s-2}^{s+2} \frac{1}{\sqrt{\left(4-(s-t)^{2}\right)\left(4 \tilde{\lambda}^{2}-t^{2}\right)}} d t,
$$


(4) if $2 \tilde{\lambda}-2<s \leq 2 \tilde{\lambda}+2$, then

$$
\rho^{(\lambda)}(s)=\pi^{-2} \int_{s-2}^{2 \tilde{\lambda}} \frac{1}{\sqrt{\left(4-(s-t)^{2}\right)\left(4 \tilde{\lambda}^{2}-t^{2}\right)}} d t .
$$

- Case 2: $0<\tilde{\lambda}<1$

(1) if $s<-2-2 \tilde{\lambda}$ or $s>2+2 \tilde{\lambda}$, then $\rho^{(\lambda)}(s)=0$,

(2) if $-2-2 \tilde{\lambda} \leq s<2 \tilde{\lambda}-2$, then

$$
\rho^{(\lambda)}(s)=\pi^{-2} \int_{-2 \tilde{\lambda}}^{s+2} \frac{1}{\sqrt{\left(4-(s-t)^{2}\right)\left(4 \tilde{\lambda}^{2}-t^{2}\right)}} d t,
$$

(3) if $2 \tilde{\lambda}-2 \leq s \leq 2-2 \tilde{\lambda}$, then

$$
\rho^{(\lambda)}(s)=\pi^{-2} \int_{-2 \tilde{\lambda}}^{2 \tilde{\lambda}} \frac{1}{\sqrt{\left(4-(s-t)^{2}\right)\left(4 \tilde{\lambda}^{2}-t^{2}\right)}} d t,
$$

(4) if $2-2 \tilde{\lambda}<s \leq 2 \tilde{\lambda}+2$, then

$$
\rho^{(\lambda)}(s)=\pi^{-2} \int_{s-2}^{2 \tilde{\lambda}} \frac{1}{\sqrt{\left(4-(s-t)^{2}\right)\left(4 \tilde{\lambda}^{2}-t^{2}\right)}} d t .
$$

All these integrals can be expressed in terms of the complete elliptic integral $K(x)$ that appeared previously in (12) and (34).

For instance, consider the case that $\tilde{\lambda}>1$ and $2 \tilde{\lambda}-2<s \leq 2 \tilde{\lambda}+2$. Then we can represent the density by

$$
\rho^{(\lambda)}(s)=\pi^{-2} \int_{s-2}^{2 \tilde{\lambda}} \frac{1}{\sqrt{\left(4-(s-t)^{2}\right)\left(4 \tilde{\lambda}^{2}-t^{2}\right)}} d t .
$$

Now consider the integral representation

$$
\frac{1}{2 \pi^{2} \sqrt{\tilde{\lambda}}} K\left(\frac{(2 \tilde{\lambda}+2)^{2}-s^{2}}{16 \tilde{\lambda}}\right)=\frac{1}{4 \pi^{2} \sqrt{\tilde{\lambda}}} \int_{-1}^{1} \frac{d x}{\sqrt{\left(1-x^{2}\right)\left(1-\frac{(2 \tilde{\lambda}+2)^{2}-s^{2}}{16 \tilde{\lambda}} x^{2}\right)}} .
$$

The rational substitution

$$
x=\frac{8 \tilde{\lambda}-8 \tilde{\lambda}^{2}-2 \tilde{\lambda} s^{2}+4 \tilde{\lambda} s t+2 t^{2}-2 \tilde{\lambda} t^{2}}{4 \tilde{\lambda}^{2} s+4 t-4 \tilde{\lambda}^{2} t-s^{2} t+s t^{2}}
$$

transforms this (as one readily checks) to the expression in (A7). Thus we have

$$
\rho^{(\lambda)}(s)=\frac{1}{2 \pi^{2} \sqrt{\tilde{\lambda}}} K\left(\frac{(2 \tilde{\lambda}+2)^{2}-s^{2}}{16 \tilde{\lambda}}\right)
$$

in this case. The other cases are treated in a similar fashion. We end up with the following general formula which holds for all positive values of $\tilde{\lambda}$ :

$$
\rho^{(\lambda)}(s)= \begin{cases}0, & |s| \geq 2+2 \tilde{\lambda}, \\ \frac{1}{2 \pi^{2} \sqrt{\tilde{\lambda}}} K\left(\frac{(2 \tilde{\lambda}+2)^{2}-s^{2}}{16 \tilde{\lambda}}\right), & |2 \tilde{\lambda}-2| \leq|s|<2 \tilde{\lambda}+2, \\ \frac{2}{\pi^{2} \sqrt{(2 \tilde{\lambda}+2)^{2}-s^{2}}} K\left(\frac{16 \tilde{\lambda}}{(2 \tilde{\lambda}+2)^{2}-s^{2}}\right), & |s|<2 \tilde{\lambda}-2 .\end{cases}
$$


We remark that the limiting case $\tilde{\lambda}=0$ yields the density of an arcsine distribution on $[-2,2]$.

\section{APPENDIX C: EQUATION (32) CAN BE OBTAINED AS IN REF. 1}

In order to reproduce from the lattice walks formulation in Ref. 1 the results obtained above for $\lambda \neq 2$, all that is needed is to consider lattice walks with asymmetric probability jumps on the horizontal axis versus the vertical axis in a ratio $\lambda$. One sets in the generating function $A\left(x_{1}, x_{2}, y_{1}\right.$, $y_{2}$, Q) [defined in Eq. (10) of Ref. 1] $x_{1} \rightarrow z x, x_{2} \rightarrow z / x, y_{1} \rightarrow(\lambda / 2) z y$, and $y_{2} \rightarrow(\lambda / 2) z / y$ and looks at the coefficients with vanishing exponents in $x$ and $y$ (i.e., $m_{1}-m_{2}=0$ and $l_{1}-l_{2}=0$ ) and exponent $n$ in $z$ (i.e., $m_{1}+m_{2}+l_{1}+l_{2}=n$ ),

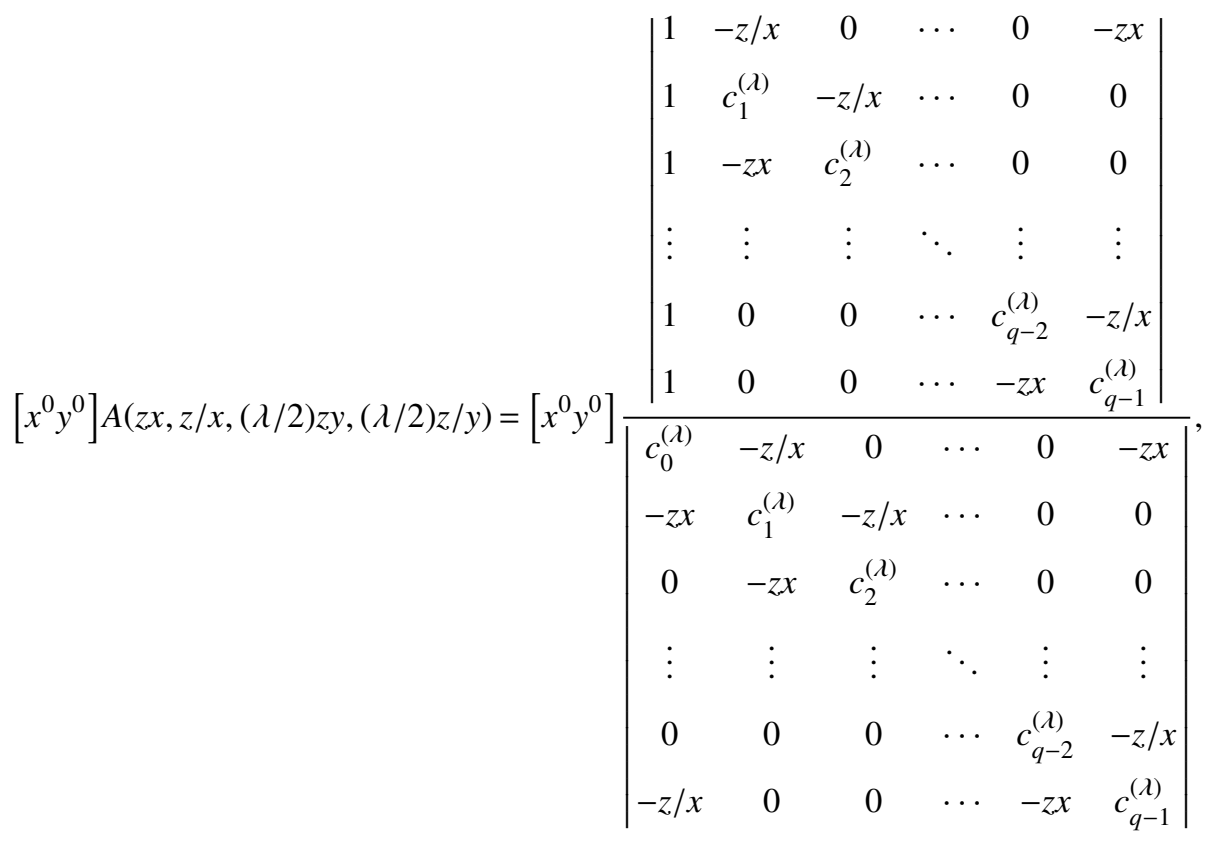

where $c_{k}^{(\lambda)}=1-(\lambda / 2) z\left(\mathrm{Q}^{k} y+\mathrm{Q}^{-k} y^{-1}\right)$ with $\mathrm{Q}=e^{2 i \pi p / q}$.

As in Ref. 1, the denominator has the form

$$
\Delta(z x, z / x,(\lambda / 2) z y,(\lambda / 2) z / y)=1-z^{q}\left(x^{q}+x^{-q}+(\lambda / 2)^{q}\left(y^{q}+y^{-q}\right)\right)+V\left(z^{2},(\lambda / 2)^{2} z^{2}\right) .
$$

For $x=y=1$, we get

$$
\Delta(z, z,(\lambda / 2) z,(\lambda / 2) z)=1-z^{q}\left(2+2(\lambda / 2)^{q}\right)+V\left(z^{2},(\lambda / 2)^{2} z^{2}\right)
$$

so that

$$
\begin{aligned}
\Delta(z x, z / x, & (\lambda / 2) z y,(\lambda / 2) z / y) \\
& =\Delta(z, z,(\lambda / 2) z,(\lambda / 2) z)+z^{q}\left(2+2(\lambda / 2)^{q}\right)-z^{q}\left(x^{q}+x^{-q}+(\lambda / 2)^{q}\left(y^{q}+y^{-q}\right)\right) .
\end{aligned}
$$

On the other hand, recall that both the polynomial $b_{p / q}^{(\lambda)}(z)=-\sum_{j=0}^{\left[\frac{q}{2}\right]} a_{p / q}^{(\lambda)}(2 j) z^{2 j}$ defined in (25) and the matrix $m_{p / q}^{(\lambda)}(e, 0,0)$ defined in (23) are related by (26). Since also

$$
\Delta(z, z,(\lambda / 2) z,(\lambda / 2) z)=(-z)^{q} \operatorname{det}\left(m_{p / q}^{(\lambda)}(1 / z, 0,0)\right),
$$

it follows that

$$
\Delta(z, z,(\lambda / 2) z,(\lambda / 2) z)=b_{p / q}^{(\lambda)}(z)-z^{q}\left(2+2(\lambda / 2)^{q}\right)
$$

and finally

$$
\Delta(z x, z / x,(\lambda / 2) z y,(\lambda / 2) z / y)=b_{p / q}^{(\lambda)}(z)-z^{q}\left(x^{q}+x^{-q}+(\lambda / 2)^{q}\left(y^{q}+y^{-q}\right)\right) .
$$


Accordingly, and as shown in the Appendix of Ref. 1 [see the proof of (18)], the numerator at order $x^{0} y^{0}$ ends up being expressed only in terms of $b_{p / q}^{(\lambda)}(z)$,

$$
\left[x^{0} y^{0}\right]\left|\begin{array}{cccccc}
1 & -z / x & 0 & \cdots & 0 & -z x \\
1 & c_{1}^{(\lambda)} & -z / x & \cdots & 0 & 0 \\
1 & -z x & c_{2}^{(\lambda)} & \cdots & 0 & 0 \\
\vdots & \vdots & \vdots & \ddots & \vdots & \vdots \\
1 & 0 & 0 & \cdots & c_{q-2}^{(\lambda)} & -z / x \\
1 & 0 & 0 & \cdots & -z x & c_{q-1}^{(\lambda)}
\end{array}\right|=b_{p / q}^{(\lambda)}(z)-\frac{z}{q} b_{p / q}^{(\lambda)}(z)^{\prime} .
$$

Finally (A8) rewrites as

$$
\begin{gathered}
{\left[x^{0} y^{0}\right] A(z x, z / x,(\lambda / 2) z y,(\lambda / 2) z / y)=\left[x^{0} y^{0}\right] \frac{b_{p / q}^{(\lambda)}(z)-\frac{z}{q} b_{p / q}^{(\lambda)}(z)^{\prime}}{b_{p / q}^{(\lambda)}(z)-z^{q}\left(x^{q}+x^{-q}+(\lambda / 2)^{q}\left(y^{q}+y^{-q}\right)\right)}} \\
=\left(1-\frac{z b_{p / q}^{(\lambda)}(z)^{\prime}}{q b_{p / q}^{(\lambda)}(z)}\right) \sum_{k \geq 0}\left(\frac{z^{q}}{b_{p / q}^{(\lambda)}(z)}\right)^{k}\left[x^{0} y^{0}\right]\left(x^{q}+x^{-q}+(\lambda / 2)^{q}\left(y^{q}+y^{-q}\right)\right)^{k}
\end{gathered}
$$

which yields the generating function (32) for the traces $\operatorname{Tr}\left(H_{2 \pi p / q}^{(\lambda)}\right)^{n}$; we have

$$
\begin{aligned}
& {\left[x^{0} y^{0}\right]\left(x^{q}+x^{-q}+(\lambda / 2)^{q}\left(y^{q}+y^{-q}\right)\right)^{k}=\left[x^{0} y^{0}\right] \sum_{k_{1}+k_{2}+k_{3}+k_{4}=k}\left(\begin{array}{c}
k \\
k_{1}, k_{2}, k_{3}, k_{4}
\end{array}\right)(\lambda / 2)^{q\left(k_{1}+k_{2}\right)} x^{q\left(k_{3}-k_{4}\right)} y^{q\left(k_{1}-k_{2}\right)}} \\
& =\sum_{\substack{k_{1}+k_{3}=k / 2 \\
k \text { even }}}\left(\begin{array}{c}
k \\
k_{1}, k_{1}, k_{3}, k_{3}
\end{array}\right)(\lambda / 2)^{2 q k_{1}} \\
& =\left(\begin{array}{c}
k \\
k / 2
\end{array}\right) \sum_{k_{1}=0}^{k / 2}\left(\begin{array}{c}
k / 2 \\
k_{1}
\end{array}\right)^{2}(\lambda / 2)^{2 q k_{1}} .
\end{aligned}
$$

The coefficient at order $x^{0} y^{0}$ of $\left(x^{q}+x^{-q}+(\lambda / 2)^{q}\left(y^{q}+y^{-q}\right)\right)^{k}$ is $\left(\begin{array}{c}k \\ k / 2\end{array}\right) \sum_{k_{1}=0}^{k / 2}\left(\begin{array}{c}k / 2 \\ k_{1}\end{array}\right)^{2}(\lambda / 2)^{2 q k_{1}}$ if $k$ is even and 0 otherwise so that finally

$$
\sum_{n \geq 0, n \text { even }} \operatorname{Tr}\left(H_{2 \pi p / q}^{(\lambda)}\right)^{n} z^{n}=\left(1-\frac{z b_{p / q}^{(\lambda)}(z)^{\prime}}{q b_{p / q}^{(\lambda)}(z)}\right) \sum_{k \geq 0}\left(\frac{z^{q}}{b_{p / q}^{(\lambda)}(z)}\right)^{2 k}\left(\begin{array}{c}
2 k \\
k
\end{array}\right) \sum_{k_{1}=0}^{k}\left(\begin{array}{c}
k \\
k_{1}
\end{array}\right)^{2}(\lambda / 2)^{2 q k_{1}} .
$$

${ }^{1}$ S. Ouvry, S. Wagner, and S. Wu, "On the algebraic area of lattice walks and the Hofstadter model," J. Phys. A: Math. Theor. 49, 495205 (2016).

${ }^{2}$ D. R. Hofstadter, "Energy levels and wave functions of Bloch electrons in rational and irrational magnetic fields," Phys. Rev. B 14(6), 2239-2249 (1976).

${ }^{3}$ C. Kreft, Explicit computation of the discriminant for the Harper equation with rational flux, SFB 288 Preprint No. 89 , 1993.

${ }^{4}$ Y. Last, "Zero measure for the almost Mathieu operator," Commun. Math. Phys. 164, 421-432 (1994).

${ }^{5}$ W. Chambers, "Linear-Network Model for Magnetic Breakdown in Two Dimensions," Phys. Rev 140, A135-A143 (1965).

${ }^{6}$ O. Lipan, "Bandwidths statistics from the eigenvalue moments for Harper-Hofstadter problem," J. Phys. A: Math. Gen. 33(39), 6875 (2000).

${ }^{7}$ The mid-band energies (13) can be obtained from Bethe-ansatz equations for the quantum group $U_{q}\left(s l_{2}\right)$. See P. B. Wiegmann and A. V. Zabrodin, Quantum group and magnetic translations. Bethe-ansatz solution for Bloch electrons in a magnetic field.

${ }^{8}$ G. H. Wannier et al., "Magnetoelectronic density of states for a model crystal," Phys. Status Solidi B 93, 337 (1979).

${ }^{9}$ S. Aubry and G. Andre, "Analyticity breaking and Anderson localization in incommensurate lattices," in Proceedings, VIII International Colloquium on Group-Theoretical Methods in Physics, Vol. 3.

${ }^{10}$ Y. Hatsuda, H. Katsura, and Y. Tachikawa, "Hofstadter's butterfly in quantum geometry,” New J. Phys. 18(10), 103023 (2016); e-print arXiv:1606.01894 [hep-th]. 